\title{
Katja Patzel-Mattern Von der Unmöglichkeit nicht zu kommunizieren
}

\section{Unternehmerische Kommunikation nach industriellen Unfällen, BASF 1921 und Hoffmann-La Roche 1976}

\begin{abstract}
The paper examines corporate communication after industrial accidents and the media reception of this communication. It compares the accident at BASF in 1921 with that of 1976 at Meda, better known as the Seveso disaster. The leading thesis is that patriarchal concepts of corporate governance marked the companies' communications in both cases. They were instrumental, alongside strategic and legal considerations, to the communicative appropriation of actions taken, as well as for public activities of the managements. However, a different configuration of the patriarchal concept of communication on the one hand and changing social conditions on the other, effected a shift in the perception of communicative action in public media. The comparison shows two things. First, successful crisis communication in the sense of social acceptance requires that concepts of order, which define the communicative behavior of companies, match those that characterize societal expectations. And second, the comparison reveals that any kind of corporate activity after industrial accidents, even public restraint, is conceived as an act of communication in a mass media society.
\end{abstract}

JEL-Codes: B 50, N 44, N 84

Keywords: Unternehmensgeschichte, Unternehmenskommunikation, Industrieunfall, chemische Industrie, BASF, Hoffmann-La Roche, business history, corporate communication, industrial accidents, chemical industry

DOI 10.1515/jbwg-2016-0018

Katja Patzel-Mattern (Prof. Dr.), Ruprecht-Karls-Universität Heidelberg, ZEGK - Historisches Seminar, Grabengasse 3-5, D-69117 Heidelberg, E-Mail: kpm@zegk.uni-heidelberg.de 
„It is one thing to hear a mighty explosion. It is another to recognize some time

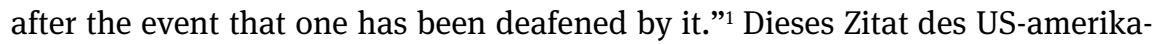
nischen Schriftstellers Norman Mailer entstammt einer Kritik des amerikanischen Engagements im damals bevorstehenden zweiten Irak-Krieg. Jenseits der konkreten politischen Bezüge verweist es auf einen grundsätzlichen Aspekt der Wahrnehmung und Beurteilung von Extremereignissen: Explosionen, Brände und Verpuffungen sind zunächst einmal Ereignisse. Erst durch ihre Wirkung auf den Menschen erhalten sie eine soziale Dimension. Der Mensch, der sich mit einem solchen Ereignis und dessen Wirkungen konfrontiert sieht, eignet es sich kommunikativ an. Der Mensch gibt dem Ereignis eine zeitliche Ordnung ebenso wie eine Sinnstruktur. Bei dem Versuch, das Ereignis zur individuellen wie kollektiven Lebenswelt in Beziehung zu setzen, konstruiert er eine Geschehensabfolge mit einem Ausgangs- und Endpunkt, die zumeist ihren Höhe- oder Wendepunkt im Ereignis selbst findet. Dies stellt die Grundlage für eine Wertung dar, ermöglicht Fragen nach Gründen, Verantwortlichkeiten und Folgen. Erst durch die kommunikative Aneignung wird das Ereignis zu einer Geschehensabfolge, die dann als Unfall oder Katastrophe sinnhaft in gesellschaftliche Kontexte eingebunden und möglicherweise auch als Symptom einer Krise interpretiert werden kann. Weder Krisen noch Katastrophen, so kann man mit dem Anglist und Literaturwissenschaftler Ansgar Nünning festhalten, sind „etwas objektiv Gegebenes oder Naturwüchsiges [...]. Vielmehr sind sie [...] als Ergebnis von Selektion, Abstraktion und Auszeichnung, mithin als diskursiv erzeugte Konstrukte, zu begreifen.“2

\section{Erkenntnisinteresse: Unternehmerische Kommunikation von Industrieunfällen}

Was für Krisen und Katastrophen im Allgemeinen gesagt werden kann, gilt auch und vielleicht besonders für entsprechende Ereignisse im Kontext industrieller Produktion. Industrieunfälle evozieren allein durch die Verflechtung des Ereignisses - der Explosion, des Brandes, der Verpuffung usw. - mit menschlichem Gestaltungswillen die Frage nach Kausalitäten. Dies fordert von Unternehmen

1 N. Mailer, Why are we at War?, New York 2003, S. 10.

2 A. Nünning: Krise als Erzählung und Metapher: Literaturwissenschaftliche Bausteine für eine Metaphorologie und Narratologie von Krisen, in: C. Meyer/K.Patzel-Mattern/G.J. Schenk (Hg.), Krisengeschichte(n). „Krise“ als Leitbegriff und Erzählmuster in kulturwissenschaftlicher Perspektive, Stuttgart 2013, S. 117-144, hier S. 125. 
eine Beteiligung an der kommunikativen Aneignung des Ereignisses. Sie werden im Interesse einer Schadensbegrenzung mit Blick auf die ökonomische Zukunft des Unternehmens zu einem zentralen Akteur in der Konstruktion von Katastrophen- oder Krisenerzählungen. Aus der Paradoxie des Zusammenhangs, der Notwendigkeit einer Beteiligung an Kausalitätskonstruktionen einerseits und dem Ziel, Legitimationsverlusten entgegenzuwirken andererseits, resultiert das Erkenntnisinteresse dieses Aufsatzes. Er fragt am Beispiel zweier Industrieunfälle nach der Positionierung der Unternehmensleitungen im öffentlichen Diskurs. Als Untersuchungsgrundlage dienen zum einen die Explosion bei der BASF in Oppau 1921 und zum anderen der Chemieunfall bei Icmesa, einer Tochtergesellschaft der zu Hoffmann-La Roche gehörenden Givaudan, in Meda nahe Seveso im Jahr 1976. Wie und wann kann der Beitrag von Unternehmen zur „kulturelle[n] Verarbeitungspraxis der Serialisierung, Kontinuierung oder kausalen Eingliederung “3 eines Ereignisses so gestaltet werden, dass ihre gesellschaftliche und ökonomische Position nicht gefährdet wird? Erkenntnisleitend soll die Annahme sein, dass Krisenkommunikation in diesem Sinne nur dann gelingt, wenn die Ordnungsvorstellungen, die prägend für das Kommunikationsverhalten des Unternehmens sind, mit jenen übereinstimmen, welche die gesellschaftlichen Erwartungen prägen. Das betrifft nicht nur verbale Äußerungen, sondern auch Handlungen ebenso wie jede Form der ausbleibenden Interaktion. Es wird zu zeigen sein, dass im Sinne des ersten Axioms von Paul Watzlawick Zurückhaltung vonseiten der Unternehmen in der Medienöffentlichkeit ebenfalls als kommunikativer Akt verstanden und als solcher interpretiert wird. ${ }^{4}$

Diesem Untersuchungsinteresse widmet sich der Aufsatz durch die vergleichende Analyse von Reden und Stellungnahmen des Vorstandsvorsitzenden der BASF, Carl Bosch, sowie des Präsidenten des Verwaltungsrates von HoffmannLa Roche, Adolf Jann. Im Falle Carl Boschs konzentriert sich die Analyse auf dessen Rede bei der zentralen Gedächtnisfeier für die insgesamt 561 Todesopfer, die zwei Tage nach der Explosion auf dem Ludwigshafener Hauptfriedhof stattfand. ${ }^{5}$ Für Adolf Jann ist die Quellenlage diffuser. Da sich kein zentrales Dokument bestimmen lässt, werden Interviews und Erklärungen herangezogen, in

3 C. Kassung: Einleitung, in: Ders. (Hrsg.), Die Unordnung der Dinge. Eine Wissens- und Mediengeschichte des Unfalls, Bielefeld 2009, S. 9-15, hier S. 9.

4 P. Watzlawick/J.H. Beavin/D.J. Jackson, Menschliche Kommunikation - Formen, Störungen, Paradoxien, Bern ${ }^{8} 1990$, Zit. S. 51: „Handeln oder Nichthandeln, Worte oder Schweigen haben alle Mitteilungscharakter: Sie beeinflussen andere und diese anderen können ihrerseits nicht nicht auf diese Kommunikation reagieren und kommunizieren damit selbst.“

5 Rede Carl Boschs bei der Trauerfeier für die Opfer des Explosionsunglücks am 21.09.1921, in: Werkszeitung der Badischen Anilin- \& Soda-Fabrik Ludwigshafen 9/10, 1921, S. 139 f. 
denen der Präsident Anfang August 1976, rund einen Monat nach dem Ereignis, erstmals öffentlich Stellung bezog. ${ }^{6}$ Die Analyse der diskursiven Dokumente folgt einer kurzen Einführung in das jeweilige Unfallgeschehen sowie den historischen Kontext.

\section{Der Unfall in Oppau 1921 und die chemische Industrie als Hoffnungsträger}

In Oppau, einer damals noch eigenständigen Gemeinde nahe Ludwigshafen am Rhein, ereignete sich am Morgen des 21. Septembers 1921 die bis dato „weltweit größte [...] Chemie-Katastrophe“. ${ }^{7}$ Das ortsansässige Stickstoffwerk der BASF wurde durch eine Explosion fast vollständig zerstört. Ganze Gebäudekomplexe verschwanden in einem Trichter von rund 100 Metern Durchmesser und ca. 20 Metern Tiefe. Doch die Zerstörung traf nicht nur das Werksgelände. Auch der Ort Oppau war nachhaltig betroffen. Rund 90 Prozent der Wohnungen waren zerstört, schätzungsweise 7.500 der insgesamt rund 9.000 Einwohner obdachlos. 1.952 Menschen wurden verletzt, 561 verloren ihr Leben. Auslöser der Explosion war die Routinesprengung eines Düngemittelgemischs, Ammonsulfatsalpeter, das im Urteil der Zeitgenossen als ungefährlich eingestuft wurde. ${ }^{8}$ An dieser Stelle sei nur kurz darauf hingewiesen, dass ein Bestandteil des Gemischs, Ammonsalpeter, im Ersten Weltkrieg zur Produktion von Sprengstoff verwendet wurde. Das bereits in der Herstellung auftretende Risiko war also wohlbekannt. So hat

6 Hier vor allem „Was der Hoffroche-Boss zum Gift-Skandal sagt...“ Exklusivinterview mit Präsident Dr. Adolf W. Jann, in: Blick, 6.08.1976, S. 3, Hoffmann-La Roche bricht das Schweigen. Präzisierungen und Klarstellungen zum Giftunglück von Seveso, in: Neue Zürcher Zeitung Nr. 187, 12.08.1976, S. 7 sowie Briefausschnitte zitiert nach T. Jungkind: Risikokultur und Störverhalten der chemischen Industrie. Gesellschaftliche Einflüsse auf das unternehmerische Handeln von Bayer und Henkel seit der zweiten Hälfte des 20. Jahrhunderts, Stuttgart 2013, S. 247-255.

7 G. Braun, Eine Großstadt wird korrigiert - Ludwigshafen in der Weimarer Republik 19191933, in: S. Mörz/K.J. Becker (Hg.), Geschichte der Stadt Ludwigshafen am Rhein, Bd. 2, Vom Ende des Ersten Weltkrieges bis zur Gegenwart, Ludwigshafen a.Rh. 2003, S. 2-182, hier S. 27. Zur Verortung im Rahmen einer Geschichte industrieller Unglücke vgl. auch J.A. Johnson, Die Macht der Synthese (1900-1925), in: W. Abelshauser (Hg.), Die BASF. Eine Unternehmensgeschichte, München 2002, S. 117-219, hier S. 209.

8 Vgl. hierzu den Bericht der Frankfurter Zeitung vom 22.09.1921, zit. nach L. Sanner, „Als wäre das Ende der Welt da“. Die Explosionskatastrophen in der BASF 1921 und 1948, Ludwigshafen 2015, S. 481-485, hier 482, vgl. aber auch ihre Ausführungen zur nachträglichen Untersuchung der Unfallursache, in: Ebda., S. 112-123. 
der Historiker Stefan Wendt für Schleswig-Holstein nachweisen können, dass während des Krieges die Unfallzahlen in der Explosivstoffbranche signifikant zunahmen. ${ }^{9}$ In der Düngemittelherstellung wurden die Eigenschaften des Endprodukts durch die Beimischung von Ammonsulfat verändert. Am Unglückstag gelang es jedoch nicht, das notwendige Mischungsverhältnis zu erreichen. 4.000 bis 4.500 Tonnen des nun explosiven Düngemittels lagerten laut eines späteren Gutachtens der BASF am Unglücksort.

Der Unfall ereignete sich in einer Branche, in der die Produktion risikobehaftet war. Unfälle - durchaus auch mit Personenschäden - passierten immer wieder. ${ }^{10}$ „Ein Großteil der Aktivitäten, die der Fabrikarbeiterverband für die Beschäftigten in der chemischen Industrie entfaltete, betraf die Sicherheitsprobleme und gesundheitlichen Risiken, die mit dieser Branche seit jeher verbunden waren." 11 Wenn auch die Auseinandersetzungen um die Betriebssicherheit durchaus kontrovers verliefen, galt die BASF doch als Hoffnungsträger in der Region und im gesamten Land. Der industriellen, großtechnischen Produktion wurde zugetraut, wesentlich zur Überwindung von Restriktionen infolge des verlorenen Krieges und der daraus resultierenden Gebietsverluste beitragen zu können. Dies galt insbesondere für die Stickstoffproduktion. War das Oppauer Werk während des Ersten Weltkriegs von zentraler Bedeutung für die Herstellung von Explosivstoffen gewesen, so versprach es nach Kriegsende die Sicherung der Ernährungslage. ${ }^{12}$ „Die Produktion künstlichen Stickstoffs ermöglichte Deutschland die Unabhängigkeit vom Import des südamerikanischen Chilesalpeters“. ${ }^{13}$ In diesem Sinne sicherte die BASF ökonomische Prosperität national, vor allem aber auch regional und lokal. Sie konnte, im Gegensatz zu anderen Unternehmen der chemischen Industrie, die Zahl ihrer Beschäftigten nach Kriegsende infolge des Ausbaus ihrer Stickstoffanlagen steigern. 1921, zum Zeitpunkt der Explosion, arbeiteten im Oppauer Werk zwischen 7.500 und 8.500

9 S. Wendt, Kalkulierbares Risiko oder programmierte Katastrophe? Nachforschungen zu den schwersten Betriebsunfällen der schleswig-holsteinischen Explosivstoffindustrie (1865-1945), in: O. Pelc (Hg.), Katastrophen in Norddeutschland. Vorbeugung, Bewältigung und Nachwirkungen vom Mittelalter bis ins 21. Jahrhundert, Wachholtz 2010, S. 213-236, hier S. 224.

10 Vgl. A. Michel, Von der Fabrikzeitung zum Führungsmittel. Werkzeitschriften industrieller Großunternehmen von 1890 bis 1945, Stuttgart 1997.

11 Braun, Großstadt, S. 26.

12 Vgl. hierzu S. Fehr, Energie für den Krieg. Schweizer Unternehmen als Zulieferer und Produzenten in der deutschen Stickstoffwirtschaft während des Ersten Weltkriegs, in: Jahrbuch für Wirtschaftsgeschichte 56, 2015, S. 479-513.

13 Sanner, Ende der Welt, hier S. 60, aber auch allgemeiner zum Diskurs über die Hoffnungen, die sich mit der Stickstoffproduktion seit der Entwicklung des Haber-Bosch-Verfahrens verbanden; Ebda. S. 60-62. 
Menschen. ${ }^{14}$ Dementsprechend resümierte ein namentlich nicht genannter Oppauer in einem Bericht der Kölnischen Volkszeit vom Unglückstag: „Mit dem gewaltigen Stickstoffwerk schnell ungeahnt groß geworden, ist Oppau auch mit ihm zusammen gestürzt und hat in weitem Bogen vieles mit ins Verderben gerissen. ${ }^{\text {" }}$ Die sich in diesem Zitat artikulierende Erschütterung des Zutrauens in den technisch-industriellen Komplex unmittelbar nach der Explosion und angesichts ihrer Zerstörungen wurde jedoch materiell-baulich recht bald überwunden. Der Wiederaufbau von Werk und Ort erfolgte an gleicher Stelle und erneut in direkter Nachbarschaft zueinander. Bereits Anfang Dezember 1921, nur elf Wochen nach der Explosion, nahm das Werk seine Produktion wieder auf und war 1923 vollständig erneuert. Der Auf- und Neubau des Ortes dauerte hingegen länger. Verantwortet durch das eigens eingerichtete Hilfswerk Oppau und finanziert durch die BASF wurde er erst Mitte der 1920er Jahre abgeschlossen. ${ }^{16}$

\section{Das Seveso-Unglück und die chemische Industrie als Zerstörerin der Natur}

Anders gestaltete sich die Lage 1976, als sich beim Chemiewerk Icmesa in Meda ein Produktionsunfall ereignete. In einem Kessel des Werkes, das Trichlorphenol (TCP), ein Zwischenprodukt der Desinfektionsmittelproduktion, herstellte, kam es am 10. Juli infolge eines Wärmestaus zu einer unkontrollierten Reaktion. Der entstehende Druck löste ein Sicherheitsventil aus; eine, wie sich herausstellen sollte, giftige Aerosolwolke trat aus und kontaminierte ein ca. 1.800 Hektar

14 Vgl. D. Schiffmann, Von der Revolution zum Neunstundentag. Arbeit und Konflikt bei der BASF 1918-1924, Frankfurt a.M. 1983, S. 460 für die niedrigere Zahl. Für die höhere Zahl vgl. C. Haller, Das Explosionsunglück in der BASF vom 21. September 1921. Katastrophenwahrnehmung und -verarbeitung in Presse, Politik und Fachwelt, in: Zeitschrift für die Geschichte des Oberrheins 161, 2013, S. 325-375, hier S. 328.

15 BASF-Archiv, Ausschnittsammlung Nachrichtenabteilung, Nr. 679: Oppau. Bisher 300 Leichen geborgen, in: Kölnische Volkszeitung, 22.09.1921.

16 Diese Zahlungen ebenso wie alle anderen Zuwendungen der BASF erfolgten stets ,auf Grund freier Vereinbarung“. Bayerisches Staatskommissariat für das Hilfswerk Oppau (Hg.), Denkschrift über die Tätigkeit des Hilfswerks Oppau, Neustadt a.d. Haardt 1925, S. IV. Nachdem der Untersuchungsbericht des Reichstages dem Unternehmen keine direkte Verantwortung für das Geschehen nachweisen konnte, war dieses juristisch nicht zu belangen. Zum Streit um die Haftungsfragen und Entschädigungszahlungen sowie die Finanzierung des Wiederaufbaus vgl. Sanner, Ende der Welt, S. 93-112. 
großes Gebiet. ${ }^{17}$ Betroffen waren neben Meda auch die Gemeinden Cesano Maderno, Desio und Seveso, das dem Unglück seinen Namen gab. Wildtiere, in erster Linie Kaninchen und Vögel, verendeten, Pflanzen verdorrten. Erst zehn Tage nach dem Unfall wurden Behörden und Öffentlichkeit darüber informiert, dass bei dem Unfall Dioxin ausgetreten war. Daraufhin wurden auf Weisung der italienischen Behörden Ende Juli und Anfang August mehr als 730 Menschen evakuiert, vergiftete Nutztiere mussten getötet werden. Erst im Oktober 1977 konnten die ersten Bewohner nach umfangreichen Dekontaminierungsarbeiten zurückkehren. ${ }^{18}$ Menschliche Todesopfer verursachte das Unglück zwar nicht unmittelbar, aber die Regierung stellte schwangeren Frauen der betroffenen Gemeinden in den ersten drei Schwangerschaftsmonaten frei, eine Abtreibung vornehmen zu lassen. ${ }^{19}$ Von schätzungsweise 150 Betroffenen stellten bis Oktober 1976 ,125 Frauen einen Antrag auf Abbruch der Schwangerschaft. In 30 Fällen wurde dieser Eingriff genehmigt und durchgeführt." ${ }^{20}$ Zahlenmäßig schwerer wog die Zahl der Chlorakne-Erkrankungen. Bis zum April 1977 wurden bei 187 Bewohnern der betroffenen Gemeinden (davon 88 Prozent Kinder) die Akne-ähnlichen Hautläsionen infolge von Vergiftung diagnostiziert. ${ }^{21}$ Entschädigungszahlungen an die Betroffenen erfolgten erst spät und nach juristischen Auseinandersetzungen. 1991 wurde Givaudan zu Schadensersatzzahlungen ,in Höhe von jeweils 2 Millionen Lire (10.033 Euro) an 21 Privatpersonen“ verurteilt. ${ }^{22}$

17 „Das ausgetretene Gemisch bestand aus Trichlorphenol, Aethylenglykol, Natriumhydroxid und geringen Mengen an Dioxid.“ Zit., Jungkind, Risikokultur, S. 543. Eine Studie aus dem Jahr 1998 schätzte die Menge ausgetretenen Dioxins auf mind. $34 \mathrm{~kg}$ : P.A. Bertazzi/I. Bernucci/G. Brambilla/D. Consonni/A.C. Pesatori, The Seveso Studies on Early and Long-Term Effects of Dioxin Exposure. A Review, in: Environmental Health Perspectives 106/Suppl. 2, 1998, S. 625633, hier S. 625.

18 Entschädigungszahlungen an die betroffenen Gemeinden erfolgten ab 1981. Sie beliefen sich auf $748.900 €$ für Desio, 1.470.000 € für Cesano Maderno, 1.470.000 € für Meda und 7,75 Millionen $€$ für Seveso. Die Zahlen sind entnommen aus: M. Hofmann, Lernen aus Katastrophen. Nach den Unfällen von Harrisburg, Seveso und Sandoz, Berlin 2008, S. 214 f.

19 Auch wird seit dem Unglück immer wieder darüber diskutiert, inwiefern sich Krebs- und Diabeteserkrankungen in der Region auf die Kontamination mit Dioxin zurückführen lassen.

20 S. Böschen, Risikogenese. Prozesse gesellschaftlicher Gefahrenwahrnehmung: FCKW, DDT, Dioxin und Ökologische Chemie, Wiesbaden 2000, S. 224.

21 P.A. Bertazzi u.a., Seveso Studies, hier S. 626. Der Artikel diskutiert auch Auswirkungen des Unglücks auf Krankheitsverläufe (Herz-Kreislauf, Krebs etc.) und Lebenserwartung und diskutiert dabei das Verhältnis von psychischen und chemischen Belastungen. S. Böschen, Risikogenese, S. 222 weist auf divergierende Zahlen zu den Chlorakne-Erkrankungen in den Jahren nach dem Unglück hin.

22 Hofmann, Lernen, S. 216. 
Es waren gerade die Bilder der erkrankten Kinder und der durch sie vermittelte Eindruck, dass die Schwächsten der Gesellschaft am stärksten unter den Folgen des Unfalls zu leiden hatten, die in der westeuropäischen und insbesondere in der deutschen Medienöffentlichkeit Aufmerksamkeit erzeugten. ${ }^{23}$ Das Foto der erkrankten, damals vierjährigen Alice Senno gab dem Geschehen nicht nur 1976 ein Gesicht, sondern fungierte auch später, anlässlich des 30. Jahrestages, in deutschen Medien als Symbol des Unfalls und seiner Folgen. ${ }^{24}$ Ähnlich nachhaltig wie die Fotografien der erkrankten Kinder wirkten auch Schilderungen des Tiersterbens. Zusammen mit Bildern von Kadavern und Menschen in Schutzanzügen, die diese einsammelten oder Bodenproben nahmen, weckten sie apokalyptische Assoziationen. „Vernichtet der Mensch seinen Lebensraum, den er zu beherrschen sich anschickte? Die Katastrophe von Seveso warf die Frage auf nach den Grenzen des Wachstums. "25 Mit diesen Worten führte der Spiegel im August 1976 einen Artikel mit dem Titel Geplünderte, vergewaltigte, vergiftete Erde ein und stellte damit zugleich einen wirkmächtigen Zusammenhang her. Vor dem Hintergrund des 1972 veröffentlichten Berichts Limits to Growth des Club of Rome und des zehn Jahre zuvor erschienenen Sachbuchs Silent Spring von Rachel Carson schien das Geschehen in Seveso alle besorgten Prognosen zu bestätigen: ${ }^{26}$ Die industrielle Produktion, so die Wahrnehmung, schuf nicht länger primär Wohlfahrt, sondern vernichtete diese. Dies galt in den Augen einer kritischen Medienöffentlichkeit insbesondere für die chemische Industrie, welche, so der eben zitierte Spiegel-Artikel weiter, „mit ihrem Pesthauch das Gleichgewicht der Natur zu zerstören droht. Damals [in ihrem Roman Silent Spring, d.Vf.] schloss die Autorin ihre Schreckensvision mit den Worten: ,Diese Stadt gibt es in Wirklichkeit nicht.' Seit dem 10. Juli, als in der norditalienischen Ortschaft Seveso eine ,weiße Wolke‘ aufstieg, gibt es sie.“27 Der Spiegel verlieh mit seinen Worten einem Vertrauensverlust Ausdruck, der nicht nur Icmesa, Givaudan oder Hoffmann-La Roche traf, sondern die chemische Industrie

23 Vgl. hierzu den Titel sowie den entsprechenden Artikel: Jetzt frisst das Gift unsere Kinder, in: Stern 52,1976.

24 Exemplarisch: Synonym für die Angst vor der Chemie, in: Süddeutsche Zeitung, SZ.de, 19.05.2010, http://www.sueddeutsche.de/panorama/jahre-seveso-synonym-fuer-die-angst-vorder-chemie-1.920972, 12.04.2016.

25 Geplünderte, vergewaltigte, vergiftete Erde, in: Der Spiegel 53,1976, S. 120-124, hier S. 120.

26 D. Meadows/D. Meadows/J. Randers/W.W. Behrens III, The Limits to Growth, New York 1972 und R.L. Carson, Silent Spring, Boston 1962.

27 Geplünderte Erde, S. 120. Die weiße Wolke bezieht sich auf ein von Carson benutztes Bild, das die Pestizide als weißes körniges Pulver, das aus der Luft verteilt wird, vorstellt. Die Aerosolwolke ebenso wie das niedergeschlagene Dioxin waren demgegenüber farblos. 
insgesamt unter Generalverdacht stellte. Der gesellschaftliche Konsens über die Gestaltung von Fortschritt durch industrielles Wachstum war vor dem Hintergrund aktueller gesellschaftlicher und wirtschaftlicher Entwicklungen, der Beteiligung und Pluralität einfordernden sozialen Bewegungen, ebenso wie der ersten Ölkrise 1973/74, brüchig geworden. Vor diesem Hintergrund vermag es nicht zu erstaunen, dass die Nachbarschaft von Werk und Wohnbevölkerung in Seveso nach der Dekontamination nicht wiederhergestellt wurde. Vielmehr wurde die Fabrik abgerissen und an der Stelle der ehemals am stärksten kontaminierten Zone A entstand mit dem Bosco delle Querce ein Naturpark.

\section{Unternehmerische Kommunikation unmittelbar nach den Unfällen}

Angesichts der geschilderten divergierenden Rahmenbedingungen 1921 und 1976 ließe sich vermuten, dass die Unternehmen BASF und Hoffmann-La Roche die jeweiligen Unfälle durchaus unterschiedlich kommunizierten. Diese Annahme scheint sich zunächst zu bestätigen, schaut man auf die Reaktionen unmittelbar nach den Ereignissen in Oppau und Seveso. So beschrieb Karl Holdermann, der Biograph Carl Boschs, die Reaktionen des Vorstandsvorsitzenden aus der Retrospektive 1960 folgendermaßen: „Carl Bosch hatte voller Entsetzen die Explosion bis nach Heidelberg gehört. [...] Er griff sofort nach dem Telefon. Der Versuch, von Heidelberg aus eine Verbindung mit dem Werk in Oppau zu bekommen, war erfolglos. Als er eben den Hörer aufgelegt hatte, rief Ernst Schwarz [Chemiker und Vertrauter Boschs] aus Ludwigshafen an und schilderte, was geschehen war. Bosch fuhr sofort nach Ludwigshafen und ließ sich dort berichten, was man bisher festgestellt hatte. In Begleitung von Schwarz fuhr er weiter nach Oppau.“"28 Folgt man den Ausführungen Karl Holdermanns weiter, so erwies sich Carl Bosch, seiner Einschätzung nach, sowohl in der Begegnung mit lokalen Politikern, als auch mit leitenden Mitarbeitern des Unternehmens als besonnener Krisenmanager, der angesichts von Zerstörung, Leid und Chaos notwendige Maßnahmen zur Überwindung der Krise einleitete und bereits am Unfalltag neue Visionen für den Wiederaufbau entwickelte. Die Stilisierung gipfelt in einer abschließenden Bewertung des Verhaltens des Vorstandsvorsitzenden: „Alles in allem gab er ein Musterbeispiel, wie der Leiter eines großen Unternehmens in einer kritischen Situation durch sein Beispiel neuen Mut und die Kraft zum Wiederaufbau und

28 K. Holdermann:, Carl Bosch, Düsseldorf 1960, S. 183. 
zur Weiterarbeit in seinen Mitarbeitern wecken konnte." ${ }^{29}$ Sicherlich sind diese Ausführungen hinsichtlich ihrer Argumentationslogik, ihres Spannungsbogens sowie der einseitigen Wertungen kritisch zu hinterfragen. Sie vermitteln weniger einen Eindruck des Handelns Carl Boschs im Jahr 1921. Vielmehr dokumentieren sie dessen nachträgliche Interpretation. Der Biograph beschrieb Carl Bosch in seinem 1960 erschienenen Buch als Wirtschaftskapitän. Er griff damit eine Vorstellung auf, die in den 1950er Jahren etabliert worden war, um dem Vorwurf, Unternehmer seien Steigbügelhalter Hitlers gewesen, entgegen zu wirken. Sie erlaubte eine Inszenierung unternehmerischer Bedeutung für das sogenannte Wirtschaftswunder über alle politischen Brüche hinweg. ${ }^{30}$ Eingedenk dieser kritischen Einordnung verweisen die Ausführungen Karl Holdermanns zugleich auch auf Merkmale des Krisenmanagements des historischen Boschs, die durch zeitgenössische Quellen belegt sind: So zeigte sich, wie das Protokoll der ersten Gemeinderatssitzung in Oppau nach der Katastrophe nahelegt, Carl Bosch unmittelbar nach dem Unglück persönlich involviert. Nach Angaben des damaligen Bürgermeisters Süß habe dieser bei einem Besuch am Unglückstag mit den Tränen zu kämpfen gehabt. ${ }^{31}$ Überdies reagierte Bosch als Verantwortlicher des Unternehmens gegenüber einer ausgewählten und zahlenmäßig begrenzten Öffentlichkeit zeitlich unmittelbar. Bereits am 21. September, dem Tag des Geschehens, sprach er mit unterschiedlichen Anspruchsgruppen - Bürgermeister, Magistrat und Betriebsleitern. Dabei ist allerdings zu berücksichtigen, dass die wichtigste Anspruchsgruppe eines Unternehmens, seine Mitarbeiterinnen und Mitarbeiter, bei der Intervention des Vorstandsvorsitzenden in der überwiegenden Zahl ebenso unberücksichtigt blieben wie die materiell oder körperlich betroffenen Opfer und die Hinterbliebenen. Auch positionierte sich Carl Bosch weder am Tag der Explosion noch in den Folgetagen medienöffentlich. Dies än-

29 Ebda., S. 185.

30 A. von Plato, Wirtschaftskapitäne. Biographische Selbstkonstruktionen von Unternehmern der Nachkriegszeit, in: A. Schildt/A. Sywottek (Hg.), Modernisierung im Wiederaufbau. Die westdeutsche Gesellschaft der 1950er Jahre, Bonn 1993, S. 377-391. Eine kritische Diskussion unterschiedlicher Arbeiten zu diesem Themenkomplex bietet, insbesondere mit Blick auf Fragen der Amerikanisierung, R. Rosenberger, Experten für Humankapital. Die Entdeckung des Personalmanagements in der Bundesrepublik Deutschland, München 2008, S. 22-26. Zu Elitenkontinuitäten über den NS hinaus siehe P. Erker/T. Pierenkemper (Hg.), Deutsche Unternehmer zwischen Kriegswirtschaft und Wiederaufbau. Studien zur Erfahrungsbildung von IndustrieEliten, München 1999.

31 Oberbürgermeisteramt an das Bezirksamt Frankenthal, 22.09.1921. StALu, Op 2478, Beilage. Auch hier ist quellenkritisch zu berücksichtigen, dass Süß diese Zuschreibung im Hinblick auf die Frage nach Entschädigungszahlungen macht und sie so durchaus auch funktional eingesetzt haben könnte. 
derte sich erst mit seiner Teilnahme an der zentralen Trauerfeier für die Opfer des Unfalls am 25. September 1921 auf dem Hauptfriedhof in Ludwigshafen. Mit der Rede, die er dort im Auftrag des Vorstands hielt, richtete er sich an die anwesenden Hinterbliebenen, die Wohnbevölkerung und die Politik auf Reichsebene, vertreten u.a. durch den ebenfalls an der Trauerfeier teilnehmenden Reichspräsidenten Friedrich Ebert. Zugleich adressierte er via Medien, welche die Worte Boschs abdruckten, die nationale Öffentlichkeit. ${ }^{32}$

Deutlich anders als Carl Bosch, der zeitnah mit Ortbesuchen und Gesprächen auf das Unfallgeschehen reagierte und mit seiner Rede vier Tage nach dem Ereignis öffentlich Stellung nahm, agierte Adolf W. Jann, der Präsident des Verwaltungsrates von Hoffmann-La Roche, nach dem Unfall in Meda. Zwar drang auch hier die Kunde des Unfalls und seines Ausmaßes rasch in die Konzernzentrale vor. So war dem Unternehmen bereits am 11. Juli bekannt, dass Dioxin ausgetreten war. Dennoch blieben nach außen gerichtete Reaktionen zunächst aus. Auch konzernintern gab es erst fünf Tage nach dem Ereignis eine erste offizielle Krisensitzung. ${ }^{33}$ Sie wurde nicht von Adolf W. Jann selbst, sondern von dessen Stellvertreter Alfred Hartmann geleitet. Jann befand sich zum Zeitpunkt des Unfalls, ebenso wie der Generaldirektor von Givaudan, Guy Waldvogel, auf einer Geschäftsreise in Lateinamerika, die beide nicht vorzeitig beendeten. Erst eine gute Woche später kehrte Jann nach Basel zurück. Die lange Abwesenheit Janns aber auch Waldvogels ließ ein Weisungs- und Entscheidungsvakuum entstehen. Darauf deuten Stellungnahmen von Funktionsträgern im Konzern hin. Jörg Sambeth, damaliger technischer Direktor der Givaudan, beschrieb in einem autobiographischen Roman, dass es unmöglich gewesen sei, die Entscheider, unter anderem den Präsidenten, zu erreichen. Sambeth berichtete zudem von der daraus resultierenden Orientierungslosigkeit. ${ }^{34}$ Auch Äußerungen des damaligen Pressesprechers bei Hoffmann-La Roche, Hans Fehr, und eine schriftliche Stellungnahme Adolf W. Janns gegenüber den Medien thematisierten Abwesen-

32 Werkszeitung, S. 139/f.

33 Vgl. Jungkind, Risikokultur, S. 249.

34 Sambeth unter Verwendung anderer Namen: „Hilfe wird er bitter benötigen. Er sieht das Chaos heraufziehen. [...] Anton ruft in der Konzernzentrale an. Dr. Nonner sei noch nicht von der Reise zurück, sagt ihm der Nachtportier. Anton will Dr. Nonners Stellvertreter, [sic] sprechen. Dr. Thurnheer sei noch nicht im Haus. [...] Dann versucht er es zum hundertsten Mal mit Armbruster. Wie zuvor hat er kein Glück.“, Zit. J. Sambeth, Zwischenfall in Seveso, Zürich 2016, S. 206 f. Im gleichen Zusammenhang auch S. 208-212, S. 230-234 und S. 238-240. Zieht man die Ausführungen Sambeths zu Rat ist allerdings zu berücksichtigen, dass die Schrift der retrospektiven Einordnung seines Handelns und einer Auseinandersetzung mit der Konzernpolitik dient. Sambeth selbst wurde Mitte der 1980er Jahre wegen Fahrlässigkeit verurteilt. 
heiten und daraus folgende Informations- und Entscheidungsdefizite. ${ }^{35}$ So schrieb Jann im November 1976 in einem Brief an den Verleger und Herausgeber einer Schweizer Wirtschaftszeitung rechtfertigend, „dass in den ersten Tagen und Wochen nach dem Unfall Unklarheiten bestanden, die zu einer gewissen Informationslücke führen mussten“. Als Begründung dafür führte er den „Zeitpunkt des Unfalls; Wochenende und Ferienzeit gewisser Personen, usw.“ an und fuhr fort: „Dazu kam, dass 11 Tage nach dem Unfall der Leiter des Werkes der Firma Icmesa S.A. bei Seveso sowie sein Stellvertreter verhaftet wurden später übrigens noch ein dritter Mitarbeiter - sodass die Firma ohne Führung war [...].“" ${ }^{36}$ Auch wenn hier deutlich wird, wie sehr Jann sich darum bemühte, Verantwortlichkeit zu delegieren, in diesem Fall an die italienischen Behörden, thematisierte er zugleich die Folgen der Abwesenheiten, wie sie nicht nur vor Ort, sondern auch in der Konzernleitung herrschten. In dieser Situation legte der Vizepräsident von Hoffmann-La Roche Alfred Hartmann bei der ersten internen Krisensitzung der Leitungskräfte fünf Tage nach dem Unfall und in Vertretung des abwesenden Adolf W. Jann das weitere Vorgehen der Konzernleitung fest. Hans Fehr, Pressesprecher bei Hoffmann-La Roche, erinnerte sich in seinen Lebenserinnerungen wie folgt an die Worte Hartmanns: „Also erstens: Die Sache wird im engsten Kreis der Icmesa gehalten; Givaudan und Roche werden nicht erwähnt. Zweitens: Dass es bei der Herstellung von Hexachlorophen passiert ist, wird mit Rücksicht auf den Fall Morhange [eine Verunreinigung von Körperpuder der Firma Morhange mit dem genannten Stoff, die 1972 Todesopfer forderte] nicht erwähnt. Drittens: Dass Dioxin gebildet wurde, wird nicht erwähnt.“"37

Auch hier ist die Reichweite der zitierten Aussage quellenkritisch zu hinterfragen. Hans Fehr hatte in seinen retrospektiven Betrachtungen durchaus ein Eigeninteresse an der Schilderung pointierter Leitungsentscheidungen, dokumentierten sie doch, wie begrenzt die Handlungsspielräume des Pressesprechers

35 „Aber Givaudan-Chef Dr. Waldvogel war in Lateinamerika unterwegs, als das Unglück passierte. Es war fürchterlich. Ich saß zwölf Stunden am Telefon und versuchte, ihn zu erreichen.“ Zit., B. Kraatz, „Die Bevölkerung hat nicht stark gelitten! Interview mit dem Seveso-Beauftragten von Hoffmann-La Roche, Dr. Reggiani, in: Dies. (Hg.), Seveso oder Wie Verantwortung zur Farce wird. Ein Lehrstück, aus dem die Großchemie nichts gelernt hat, Hamburg 1979, S. 57-70, hier S. 62.

36 Schreiben von Dr. Jann an Herrn Isler/Verleger und Heraussgeber der „FINANZ UND WIRTSCHAFT“ vom 09.11.1976, in: HAR TI.8.8.1.-101570a, zit. nach Jungkind, Risikokultur, S. 252.

37 H. Fehr, Eindrücke, Basel 1995, S. 76. Der Verweis auf den Fall Morhange bezieht sich auf die Beimischung von Hexachlorophen zu einem Körperpuder der Firma Morhange im Jahr 1972. In Folge dessen starben 36 Säuglinge und mehr als 160 Kinder trugen Behinderungen davon. Vgl. Stichwort Hexachlorophen, in: K. Lohs/P. Elstner/U. Stephan (Hg.), Fachlexikon Toxikologie, Heidelberg ${ }^{4} 2009$, S. 213/214, hier S. 214. Der Unfall rief ein breites Medienecho hervor. 
waren. Auf diese Weise konnte eigenes (Nicht-)Handeln rhetorisch entschuldet werden. Dennoch gilt auch für diese zeitlich und situativ gebundenen Ausführungen, ebenso wie für jene des Bosch-Biographen Karl Holdermann, dass sie durchaus auf grundsätzliche Merkmale der Informationspolitik, diesmal von Hoffmann-La Roche nach dem Unfall 1976, verweisen: eine gegenüber der Öffentlichkeit (und gegenüber den italienischen Behörden) zeitlich verzögerte, inhaltlich zögerliche und unvollständige Kommunikation. Sie zielte darauf ab, sowohl die Involviertheit des Konzerns als auch das Ausmaß des Unfalls nicht preiszugeben. Offizielle Firmenvertreter versuchten noch am 19. September 1978, also mehr als zwei Jahre nach dem Unfall, das Agieren Hoffmann-La Roches mit einer spezifischen Organisationsform des Unternehmens zu erklären. Auf die Frage, warum der Konzern erst mit fünftägiger Verspätung den Leiter der klinischen Forschungsabteilung als vermeintlichen Fachmann nach Seveso schickte, führte der bereits zitierte Pressesprecher Hans Fehr in einem Interview aus: „Jede eigene Sparte arbeitet unabhängig, fast wie eine autonome Firmengruppe, die nur kapitalmäßig mit Roche verbunden ist. Es bestand eigentlich auch wissenschaftlich nur wenig Beziehung zwischen uns und der Tochterfirma Givaudan. Givaudan hatte ihre eigene Forschung und wurde auch sonst von La Roche in der Firmenführung nicht beeinflusst. “38 Zieht man als weitere Aussage ein internes Schreiben vom September 1976 zur Verhandlungsstrategie in den Prozessen hinzu, dann verdichtet sich das Bild. Hoffmann-La Roche arbeitete aktiv daran, Bezüge zum Mutterkonzern zu negieren. ${ }^{39}$ Die Besitzstrukturen wurden in diesem Zusammenhang als Argument genutzt - ein Argument, das die BASF 1921 so nicht nutzen konnte. Die Produktionsanlage in Oppau war in direktem Besitz des Unternehmens.

Ihren wohl sinnfälligsten Ausdruck fand die von der Betonung getrennter Verantwortung geleitete Informationspolitik Hoffmann-La Roches in der Tatsache, dass das Unternehmen erst am 11. August 1976, also einen Monat nach dem Unfall, die erste Pressekonferenz zum Thema veranstaltete und damit gegenüber einer Medienöffentlichkeit Stellung nahm. ${ }^{40}$ Nur wenige Tage zuvor war Adolf

38 Kraatz, Bevölkerung, S. 62.

39 Welche Rolle rechtliche Erwägungen dabei spielten, verdeutlicht das an Jann gerichtete Schreiben von September 1976: „Man muss in den Prozessen wie in den Verhandlungen bedacht sein, [...] Icmesa vorerst allein auftreten zu lassen, höchstens ev. noch Givaudan, wobei aber Givaudan und sicher Roche wenn irgend möglich nur als Garanten für den gesetzlich zu bestimmenden Schadensersatz auftreten sollen und nicht als Partei.“ Schreiben Dr. R. Graffenried an Dr. A. Jann et al. betreffend Stand Seveso am 08.09.und Besprechung in Lugano gleichentags vom 09. September 1976, HAR TI.8.8.1-101570a, zit. nach Jungkind, Risikokultur, S. 250 f. 40 Neue Zürcher Zeitung, Hoffmann La-Roche. 
W. Jann mit einem Exklusivinterview im Blick erstmals an die Öffentlichkeit getreten.$^{41}$ Die strategischen Motive, die erste öffentliche Stellungnahme des Präsidenten in einer Boulevardzeitung zu platzieren, lassen sich anhand der Quellenlage nicht eindeutig rekonstruieren. Möglicherweise waren sie Teil einer, wie Thilo Jungkind urteilt, „,haotischen Informationspolitik, die durch wenig Strategie und Berechenbarkeit gekennzeichnet war" ${ }^{42}$ Ebenso ist aber denkbar, dass man sich von der populären, sprachlich verdichteten und wenig Hintergrundwissen reflektierenden Präsentation erhoffte, einer vertiefenden Diskussion der Zusammenhänge zu entgehen. Eine solche Annahme wird durch die zeitliche Reihung von Interview und Pressekonferenz sowie die abgedruckten Äußerungen Janns bestärkt, die „in lockerem Plauderton“ diskussionswürdige Kausalitäten konstruierten. ${ }^{43}$ Auf sie wird im Folgenden näher eingegangen.

\section{Kommunikatives Krisenmanagement unmittelbar nach den Unfällen im Vergleich}

Der direkte Vergleich der Kommunikation vonseiten der Unternehmensleitungen verweist auf grundlegende Unterschiede. Sowohl hinsichtlich der Terminierung von Stellungnahmen und damit auch der Anlässe, die sie zum Ausgangspunkt unternehmerischer Kommunikation machten, unterschied sich das Krisenmanagement von Carl Bosch und Adolf W. Jann. Stellte einerseits der Unfall selbst den Auslöser der Kommunikation dar, so war es andererseits die Notwendigkeit, interne Verfahrensweisen nach einer Phase der Vakanz festzulegen bzw. externen Anfragen und einer zunehmenden internationalen Kritik an der fehlenden Informationspolitik entgegenzutreten. Weitere Differenzen traten hinzu. So wählte Adolf W. Jann ausschließlich die Medien als Kommunikationsvermittler, während Carl Bosch mit Gespräch und Rede auf Formen persönlicher Ansprache setzte. Daraus resultierten unterschiedliche Adressaten: Jann wandte sich an die Medienöffentlichkeit, während Carl Bosch die Anspruchsgruppen Mitarbeiter, Opfer und Hinterbliebene sowie die (Lokal-)Politik adressierte.

Dies hatte mehrere Ursachen. Eine erste liegt in den bereits erwähnten Besitzstrukturen begründet, die im Falle Hoffmann-La Roches eine Distanzierung des Mutterkonzerns legitimieren sollten. Sie fand in der Wahl unpersönlicher,

41 Blick, Hoffroche-Boss.

42 Jungkind, Risikokultur, S. 249.

43 Das meint Blick, in: Blick, 6.08.1976, S. 4. 
massenmedialer Kommunikationskanäle ihren Ausdruck. Wesentlich wichtiger aber dürften zwei weitere Aspekte sein: Veränderungen im Medienbereich und die Unterschiedlichkeit der Unfälle bei der BASF und bei Icmesa. Beide Aspekte hängen eng zusammen, weshalb sie auch gemeinsam besprochen werden sollen. Ausgangspunkt bilden die Veränderungen im Medienbereich: Zwar waren auch die 1920er Jahre im Printbereich bereits von Massenmedien geprägt, doch war die Geschwindigkeit, mit der Informationen zirkulierten, ebenso begrenzt wie das Korrespondentennetz. ${ }^{44}$ Dies brachte es mit sich, dass im Falle der medialen Kommunikation des Oppauer Explosionsunfalls wenige Berichte überregional tätiger Korrespondenten resp. Zeitungen oder Agenturmeldungen zur Referenz für die nationale deutsche Berichterstattung in der Fläche gerade unmittelbar nach dem Ereignis wurden. ${ }^{45}$ Für die unternehmensseitige Kommunikation bedeutete dies zweierlei: Zum einen konnte Carl Bosch sicher sein, dass seine Rede, sogar im wörtlichen Zitat, Verbreitung finden würde. Eine direkte Ansprache der Medien war vor diesem Hintergrund nicht dringlich und wurde überdies innerhalb eines gesellschaftlich akzeptierten patriarchalen Ordnungsdenkens auch nicht primär erwartet, wie im Folgenden noch genauer zu explizieren sein wird. Zum anderen ermöglichten es die gesellschaftlichen Erwartungshaltungen ebenso wie die oben beschriebene Struktur des Nachrichtenwesens dem Vorstandsvorsitzenden der BASF auch, sich ohne direkte Medienbeobachtung und damit auch ohne eine öffentliche Dokumentation seines Reden und Handelns - was im Hinblick auf eine spätere juristische Aufarbeitung durchaus relevant sein kann - am Unglückstag vor Ort zu bewegen.

Dies galt, obwohl die Explosion, die weithin spürbar war und eindrücklich wirkte, unmittelbar auf ein großes Medieninteresse stieß. Bereits am Unfalltag selbst wurde deutschlandweit über das Geschehen berichtet. Aufgrund der unsicheren Nachrichtenlage verstanden sich die Zeitungen in dieser Situation aber vor allem als Informationsvermittler. Sie stellten verfügbare Informationen unterschiedlicher Provenienz (Korrespondentenberichte, Augenzeugenberichte, Agenturmeldungen) mit durchaus widersprüchlichen Aussagen unkommentiert

44 Zur Bedeutung von Schnelligkeit und Unmittelbarkeit bei der Profilierung im Printbereich in den 1920er Jahren gerade auch angesichts neuer Konkurrenz durch den sich etablierenden Rundfunk vgl. U. Heitiger, Vom Zeitzeichen zum politischen Führungsmittel. Entwicklungstendenzen und Strukturen der Nachrichtenprogramme des Rundfunks in der Weimarer Republik 1923-1932, Münster 1998, hier S. 28-33.

45 Vgl. hierzu K. Patzel-Mattern, „Unsagbares Grauen“. Erzählmuster der Medienberichterstattung über die Explosionsunglücke bei der BASF 1921 und 1948, in: Dies./C. Meyer/G.J. Schenk (Hg.), Krisengeschichte(n). „Krise“ als Leitbegriff und Erzählmuster in kulturwissenschaftlicher Perspektive, Stuttgart 2013, S. 250-280, hier S. 256-258. 
nebeneinander. ${ }^{46}$ Die Berichterstattung unmittelbar nach dem Unglück war wesentlich von der Ordnung des sichtbaren Was des Unfalls und der Klärung des Wie und damit des Ereignishergangs sowie seiner nationalen Kontextualisierung geprägt. „Im geteilten und gemeinsam getragenen Leid, so eine den Pressetexten [...] eingeschriebene Überlegung, zeige sich die Zusammengehörigkeit der Nation [...].“" Kritische Fragen nach Verantwortlichkeiten traten demgegenüber in den Hintergrund. Sie wurden neben französischen und angloamerikanischen Blättern vor allem von der kommunistischen Presse in Deutschland gestellt. ${ }^{48}$ Grund dafür war die „Fraktionierung der Zeitungspresse“ ${ }^{49}$ in der Weimarer Republik; die Zeitungen orientierten sich am Spektrum der politischen Parteien. Da zum Zeitpunkt des Explosionsunfalls in Oppau die Regierung, das Kabinett Wirth II, von einer Koalition aus SPD, Zentrum und DDP getragen wurde, waren die diesem Spektrum zuzuordnenden Blätter, zu denen überregionale Tageszeitungen wie die Vossische und die Frankfurter Zeitung, aber auch die Kölnische Volkszeitung ebenso wie der sozialdemokratische Vorwärts gehörten, durch Loyalitätsbeziehungen gebunden. Auch das gab Carl Bosch informationspolitische Freiheiten. Sie wurden von der französischen Besatzung hinsichtlich der Aufklärung der Explosionsursachen durch das Unternehmen im Sinne einer bewussten Nichtinformation gedeutet: „En résumé, la Direction de la B.A.S.F. se montre très sobre de renseignements, et on reste entièrement dans la domiane de l'hypothèse." 50

Bei dem Unfall 1976 bei Icmesa stellte sich die Situation grundsätzlich anders dar. Die Zirkulation von Informationen hatte sich durch die Etablierung des Agenturjournalismus, die flächendeckende Einführung des Telefons und dann vor allem die Etablierung der Direktübertragung nachhaltig verändert. Informa-

46 Zit., Ebda., S. 259: „Es lässt sich feststellen, dass die Berichterstattung der ersten 24 Stunden 1921 weitgehend auf ungesicherten Angaben oder gar Vermutungen beruhte und zum Teil parallel, zum Teil einander folgend einander widersprechende Informationen kommunizierte. Diese werden sprachlich nicht vermittelt. Der Kommentar als eigenständige journalistische Form fehlt und auch der Hintergrundbericht spielt nur eine geringe Rolle.“

47 Ebda., S. 274.

$48 \mathrm{Im}$ Gegensatz zu anderen Medien der Arbeiterbewegung war die Rote Fahne als Organ der KPD durch die grundsätzliche Oppositionsstellung der Partei nicht durch Loyalitäten gebunden. Vgl. K. Dussel, Deutsche Tagespresse im 19. und 20. Jahrhundert, Münster 22011, hier S. 140. Die ausländische Presse thematisierte vor allem Bezüge zur Waffenproduktion im Ersten Weltkrieg und einen möglichen Verstoß gegen Auflagen des Versailler Vertrags; Sanner, Ende der Welt, S. 130-135.

49 J. Wilke, Grundzüge der Medien- und Kommunikationsgeschichte, Köln 2008, hier S. 346.

50 Compte rendu du Colonel Mouveaux, 8.10.1921. ANP, AJ/9/4328, zit. nach Sanner, Ende der Welt, S. 135. 
tionen und mit ihnen auch Bilder waren nun quasi in Echtzeit und ortsunabhängig verfügbar. ${ }^{51}$ Aktualität und Visualität wurden zu wichtigen Kriterien der Berichterstattung. Das hatte Auswirkungen auf die Kommunikation des Geschehens in Meda, da der Unfall mit dem Austritt der zwar dioxinhaltigen, aber nicht sichtbaren Aerosolwolke wenig anschaulich war. Die Journalistin Birgit Kraatz, Italienkorrespondentin verschiedener Zeitschriften sowie des ZDF, beschrieb ihren Eindruck bei der Ankunft am Unfallort am 14. Juli 1976 mit den folgenden Worten: „In Seveso schien nichts geschehen zu sein. Vor dem Fabriktor der ICMESA standen Arbeiter müßig herum und führten uns Reporter selbst auf das Fabrikgelände, das zu diesem Zeitpunkt noch unversiegelt war." ${ }^{52}$ Die scheinbare Alläglichkeit des Unfallortes, der zunächst weder durch großflächige Zerstörungen noch durch leidende Opfer gekennzeichnet war, senkte den Nachrichtenwert des Ereignisses. „Der graue Schornstein der Fabrikhalle B, eine Außenaufnahme der ICMESA, ein paar Einstellungen der Nachbarhäuser, die Giftfabrik und die Arbeiter am Eingang reichten nicht aus, um die Kollegen in der HeuteRedaktion des ZDF auf eine der größten Umweltkatastrophen des Industriezeitalters neugierig zu machen." ${ }^{53}$ Was Birgit Kraatz hier für das deutsche Fernsehen beschrieb, galt, Lokalzeitungen ausgenommen, für die Medien insgesamt. ${ }^{54}$ Ihr geringes Interesse an dem Unfall schien sich gerade in den Anfangstagen mit der zögerlichen Öffentlichkeits- und Informationspolitik Hoffmann-La Roches, aber auch der italienischen Behörden zu decken. Stellungnahmen von Verantwortlichen wurden nicht nachdrücklich angefragt. In diesem Sinne war die kommunikative Zurückhaltung der Konzernleitung zwar keinesfalls verantwortlich, aber doch unternehmens- und kommunikationspolitisch rational. Diese unausgesprochene Übereinkunft einer geringen Kommunikationswürdigkeit des Unfalls und seiner Folgen endete, als Fälle von Chlorakne bekannt wurden. Die versehrten Menschen, dann auch die verendeten Tiere, die Desinfektionskommandos und schließlich die Abriegelung und Evakuierung der verseuchten Gebiete produzierten medienwirksame Bilder. ${ }^{55}$ Ihre emotionalisierende Wirkung

51 Gerade hinsichtlich der Bilder liegt hier ein zentraler Unterschied zur Situation 1921, als Erfahrungsberichte die „noch weitgehend bildlosen Pressetexte“ illustrierten; Patzel-Mattern, Unsagbares Grauen, S. 260.

52 B. Kraatz, Reporter in Seveso, in: Dies. (Hg.), Seveso, S. 7-12, hier S. 7.

53 Ebda., S. 8

54 Vgl. hierzu H.M. Kepplinger, Aufklärung oder Irreführung? Die Darstellung von Technikfolgen in der Presse 1865-1986, in: J. Krüger/S. Ruß-Mohl (Hg.), Risikokommunikation. Technikakzeptanz, Medien und Kommunikationsrisiken, Berlin 1991, S. 109-143, hier S. 134.

55 Die Vielstimmigkeit der Berichterstattung und die Wirkmacht der kommunizierten Bilder ist eindrücklich festgehalten in dem Film von S. Gisiger, Gambit, Deutschland/Schweiz 2005. 
ließ, wie der Sozialwissenschaftler Matthias Hofmann konstatierte, Seveso „zum Symbol für die Fehlentwicklungen der Chemiepolitik an sich [werden] und wurde mit einer grundsätzlichen Diskussion des Fortschrittsparadigmas verbunden. “56 Hoffmann-La Roche als Mutterkonzern des Unfallwerks, rückte damit ins Zentrum des medienöffentlichen Interesses. Die Kritik, die in den Folgejahren auf die gesamte chemische Industrie übertragen werden sollte, konzentrierte sich zunächst auf das Basler Unternehmen. ${ }^{57}$ Angesichts dessen und vor dem Hintergrund, dass die Konzernleitung zuvor nicht aktiv geworden war, war für Adolf W. Jann Anfang August 1976 nur noch eine massenmediale Positionierung des Unternehmens im Sinne einer Kommunikation mit größtmöglicher Reichweite möglich.

\section{Unternehmenskommunikation im patriarchalen Modell}

Können Besitzverhältnisse, Entwicklungen der Mediengesellschaft und die verschiedenen Wirkungen von Explosion und Kontamination erklären, warum sich das kommunikative Krisenmanagement der BASF mit Carl Bosch an der Spitze einerseits und Hoffmann-La Roches unter der Leitung von Adolf W. Jann andererseits unterschieden, so soll dies nicht den Blick auf Gemeinsamkeiten verstellen. Sie fußten letztlich auf einem geteilten Ordnungsverständnis - dem Patriarchalismus. Im Folgenden wird aufzuzeigen sein, inwiefern dieses Ordnungsverständnis das kommunikative Handeln der Unternehmensleitungen prägte, vor allem aber, wie die unterschiedlichen Interpretationen dieses Ordnungskonzepts durch die beteiligten Akteure Kommunikationsstrategien beeinflussten.

Begriff und Bedeutung des Patriarchalismus sind in der Wirtschaft- und Unternehmensgeschichte ausführlich diskutiert worden..$^{58}$ Die ertragreichen Debatten haben gezeigt, dass es kaum möglich ist, ein geschlossenes Regelwerk zu definieren. Vielmehr gewann der Patriarchalismus seine Bedeutung für die Unter-

56 Hofmann, Lernen, S. 262. Zu Seveso als Symbol vgl. auch K. Patzel-Mattern, Industrielle Katastrophen als Ordnungselemente, in: Ordnung \& Chaos, Ruperto Carola Forschungsmagazin 3, 2013, S. 68-73, hier S. 72.

57 Die Wirkungen des Seveso-Unfalls auf die chemische Industrie arbeitet Jungkind am Beispiel Bayers und Henkels heraus; Jungkind, Risikokultur, S. 255-295.

58 Exemplarisch T. Pierenkemper, Unternehmensgeschichte. Eine Einführung in ihre Methoden und Ergebnisse, Stuttgart 2000, hier insbesondere Kap. II oder A. Nieberding, Unternehmenskultur im Kaiserreich. J..M. Voith und die Farbenfabriken vorm. Friedr. Bayer \& Co., München 2003, hier zusammenführend Kap. VI. 
nehmensführung gerade aus der Tatsache, dass das Konzept in der betrieblichen Praxis sehr unterschiedlich gefüllt werden konnte..$^{59}$ Dies ermöglichte über lange Zeiträume und grundlegende strukturelle Veränderungen hinweg eine Bezugnahme von Unternehmern auf das Konzept. Am Beispiel Carl Arnolds, Oberingenieur der Gelsenkirchener Bergwerks-A.G. und Direktor des Deutschen Instituts für Arbeitsschulung hat der Historiker Albrecht Franz aufgezeigt, wie in den 1920er Jahren überkommene „Herr im Hause“-Standpunkte und Familien-Analogien zu harmonisierenden Konzepten der Werksgemeinschaft weiterentwickelt wurden. Dies erlaubte in veränderten soziopolitischen Kontexten ein Festhalten an der Vorstellung, dass all jene, die nicht unmittelbar zum Unternehmen gehörten, nicht in dessen Entscheidungsfindung einzubeziehen seien. ${ }^{60}$

In diesem Sinne agierte auch Carl Bosch nach dem Unfall, als er gezielt die leitenden Angestellten, Opfer und Hinterbliebenen ansprach und so die Werksgemeinschaft rhetorisch konstruierte. Er tat dies im Gespräch und in der Trauerrede nicht nur der Form nach, sondern auch auf inhaltlicher Ebene. So erwähnte er in seiner Trauerrede immer da, wo er vom Aufbau und Betrieb des Werkes, der Arbeit an und in ihm sprach, explizit die Mitarbeiter als Kollektivsingular der großen Zahl. Dies gipfelte gegen Ende der Rede in der Aussage, dass er selbst angesichts der neuen Aufgabe eines Wiederaufbaus des zerstörten Werkes verzweifeln müsste, wenn er nicht sicher wäre, dass das Vertrauen der Mitarbeiter „trotz der Erschütterung durch das Unglück“ geblieben sei. ${ }^{61}$ Nur auf diesem Fundament innerer Geschlossenheit könne der Wiederaufbau erfolgen. Carl Bosch betonte hier die Werksgemeinschaft als zentralen Akteur für die Ausrichtung des unternehmerischen Handelns, aber auch für die Bewältigung der materiellen wie der menschlichen Verluste. Damit wies er innerhalb dieses Ordnungsdenkens zugleich eine mögliche öffentlich geäußerte Kritik durch Mitarbeiterinnen und Mitarbeiter als inadäquat zurück. Innerhalb des Patriarchalismus wurde deren Loyalität als Gegenleistung für unternehmerische Fürsorge erwartet.

Die Einbeziehung lokaler Politik in die Kommunikation Carl Boschs kann vor diesem Hintergrund als funktionale Erweiterung der Werksgemeinschaft interpretiert werden. Der Ort Oppau, dem für die Deckung des Arbeitskräftebedarfs des Werkes eine wichtige Rolle zukam, war durch die Explosion nahezu vollständig zerstört worden. Der lokale Umgang von politischen und administrativen Entscheidungsträgern mit dem Unfall und seine Bewältigung vor Ort waren

59 Überzeugend dargelegt bei A. Franz, Kooperation statt Klassenkampf? Zu Bedeutung kooperativer wirtschaftlicher Leitbilder für die Arbeitszeitsenkung in Kaiserreich und Bundesrepublik, Stuttgart 2014, hier S. 62 aber grundsätzlich auch Kap. 2.2 u. 2.3.

60 Ebda., S. 63/64.

61 Werkszeitung, Rede S. 140. 
somit für die Zukunft des Standortes von strategischer Bedeutung. Dies galt insbesondere auch mit Blick auf Haftungsfragen. Der Oppauer Gemeinderat hatte bei seiner ersten Sitzung unmittelbar nach dem Unfall beschlossen, beim Bezirksamt Frankenthal zu beantragen, „dass das Privatvermögen sämtlicher Direktoren und Aktionäre der Bad. Anilin- und Sodafabrik mit Beschlag belegt“ werden sollte. ${ }^{62}$ Der Umsetzung eines solches Anliegens galt es unternehmensseitig mit Blick auf eine Bestandsicherung entgegenzuwirken. Insofern kann das kommunikative Handeln Carl Boschs angesichts des Unglücks 1921 sowohl als „betriebliches Steuerungsinstrument“ mit „Koordinations-, Integrationsund Motivationsfunktionen" ${ }^{63}$ als auch als Ausdruck einer patriarchalen Unternehmenskultur verstanden werden. Mit Hartmut Berghoff ist festzuhalten, dass dieser Patriarchalismus bei der BASF in den frühen 1920er Jahren noch als ein Referenzmodell fungierte, das Unternehmensleitung wie Belegschaft integrierte und gesamtgesellschaftlich geteilt wurde. Kritik an der vergemeinschaftenden Rhetorik Boschs formulierten die Medien nach der Trauerfeier nicht. Sie wurde vielmehr unkommentiert weitergegeben. Dennoch ist zu konstatieren, dass das Leitbild des Patriarchalismus angesichts einer anonymisierten, managergeführten Leitungsstruktur im Großunternehmen BASF und soziopolitischer Umbrüche 1921 bereits an Verbindlichkeit verloren hatte. Exemplarisch sei hier auf eine Veröffentlichung hingewiesen, mit welcher die Rote Fahne, Parteizeitung der KPD, am 26. September 1921 die Stellungnahme der Unternehmensleitung zum Unfallhergang bewertete: „Es war [nach einer Erklärung der Badischen Anilinund Sodafabrik] nicht bekannt und in höchstem Maße überraschend, daß diesem Produkt Explosionsfähigkeit innewohnt. Die Ursache der Explosion wird sich kaum sicher ermitteln lassen, weil sämtliche zur Zeit der Explosion in diesen Bauten beschäftigten Personen der Katastrophe zum Opfer gefallen sind. [...] Diese Erklärung scheint darauf auszugehen, den Sachbestand zu verdunkeln. [...] Der Arbeiterrat von Oppau, die Organe der Arbeiter, müssen die Untersuchung durchführen.“ " ${ }^{64}$ Hier wurde die Idee der Werksgemeinschaft unter Rekurs auf das Konzept des Klassenkampfes infrage gestellt. Das Integrationspotential des Patriarchalismus und seine Orientierungsfunktion waren voraussetzungsreich geworden und mussten durch kommunikatives Handeln nach dem Unglück aktiv wieder neu hergestellt werden. In diesem Sinne erscheint das kommunikative Krisenmanagement der BASF strategisch. Rhetorisch einerseits integrativ,

62 Auszug aus dem Protokollbuch des Gemeinderates, Sitzung vom 21.09.1921, Stadtarchiv Ludwigshafen, Op 1903.

$63 \mathrm{H}$. Berghoff, Unternehmenskultur und Herrschaftstechnik. Industrieller Paternalismus: Hohner von 1857 bis 1918, in: GG 2, 1997, S. 167-204, hier S. 173.

64 Die Oppauer Katastrophe, in: Die Rote Fahne, 26.09.1921. 
nutzte es andererseits das hierarchische Verständnis und die „Herr im Hause“Vorstellungen des Patriarchalismus für eine restriktive Kommunikationspolitik. Diese schloss nicht nur Dritte von Informationen aus, wie das oben ausgeführte Beispiel der französischen Besatzungsmacht verdeutlicht. Vielmehr fand auch die Werksgemeinschaft bei der Kommunikation der Unfallursachen ihre Grenzen. Sie wurden, wie der Historiker Alexander Michel herausgearbeitet hat, in der innerbetrieblichen Werkszeitung nicht thematisiert. ${ }^{65}$

1976 war der Patriarchalismus als gesellschaftliche Ordnungsvorstellung durch partizipative Konzepte abgelöst worden. ${ }^{66}$ Damit hatte er seine integrative Leitfunktion weitestgehend verloren. An die Stelle der Werksgemeinschaft als einer in sich geschlossenen, auf das Unternehmen beschränkten Einheit trat ein Verständnis des Betriebs als politischer und gesellschaftlicher Ort. Betriebliche Arbeits- und Sozialbeziehungen wurden nunmehr als integrativer Bestandteil gesellschaftlicher Ordnung aufgefasst und Transparenz damit zu einer wichtigen Anforderung. ${ }^{67}$ Ungeachtet dieser Wandlungsprozesse dokumentiert das Kommunikationsverhalten Hoffmann-La Roches dennoch die Beharrungskraft patriarchaler Vorstellungen auf der Mikroebene des Unternehmens. Adolf W. Jann explizierte sein traditionelles Kommunikationsverständnis in einem Schreiben an den Verleger und Herausgeber der Schweizer Wirtschaftszeitung Finanz und Wirtschaft vom 9. November 1976: „Der Unternehmer hat zweifellos eine Informationsund Erläuterungspflicht. Er muss seine Sache in der Öffentlichkeit vertreten können. Alle diese Auseinandersetzungen werden aber nutz- und sinnlos, wenn sich Führungskräfte mehr und mehr mit der Oeffentlichkeit, [...] wie sie ihnen in der [sic] systematisch organisierten und manipulierten Massenmedien entgegentritt, auseinandersetzen müssen und dadurch ihre Führungsaufgaben vernachlässigen. Der Platz des Unternehmers ist das Unternehmen. "68 Jann rekurrierte hier auf eine Vorstellung vom Unternehmen als in sich geschlossene Einheit mit einer hierarchischen Leitungsstruktur, wie dies ja auch bei Carl

65 A. Michel, Von der Fabrikzeitung zum Führungsmittel. Werkzeitschriften industrieller Großunternehmen von 1890 bis 1945, Stuttgart 1997, S. 139-141.

66 Exemplarisch sei hier auf die seit den 1960er Jahren europaweit durchgeführten Programme zur Demokratisierung und Humanisierung der Arbeit hingewiesen, die, unter Berücksichtigung ihrer ökonomischen Funktion in wettbewerblichen Kontexten, die Wirkmächtigkeit solcher partizipativen Konzepte in ihrer Anlage und ihrem Labeling verdeutlichen.

67 Vgl. Franz, Kooperation, S. 65-69, hier mit Blick auf die Herausbildung sozialpartnerschaftlichen Ordnungsdenkens in der jungen Bundesrepublik.

68 Schreiben Dr. Jann an A. Isler, Verleger und Herausgeber der „FINANZ UND WIRTSCHAFT“ betreffend Äußerung zum Artikel von P. Rothenhäusler „Von der Katastrophen-PR zur PRKatastrophe“, Öffentlichkeitsarbeit bei Krisensituationen“ vom 9.11.1976, HAR TI.8.8.1-101570a, zit. nach Jungkind, Risikokultur, S. 254. 
Bosch bezüglich bestimmter Themen feststellbar war. Er betonte damit einseitig die Herrschaftsfunktion patriarchaler Vorstellungen und damit den Herrschaftsanspruch des Unternehmensleiters. Dies wird auch an einer weiteren Stelle in dem genannten Schreiben deutlich, wenn Jann die Informationspolitik vor Ort, wie bereits oben ausgeführt, u.a. damit begründete, dass Icmesa durch Verhaftungen ohne Führung gewesen sei. ${ }^{69}$ Demgegenüber traten Aspekte des „Geben[s] und Nehmen[s] aller Betriebsangehörigen“70 und damit auch die Fürsorgefunktion der patriarchalen Unternehmensleitung in den Hintergrund. Diese Sicht schlug sich in der zeitlichen Organisation der Kommunikation, aber auch in inhaltlichen Schwerpunktsetzungen nieder und erschien angesichts der beschriebenen Wandlungsprozesse der Medienöffentlichkeit in hohem Maße diskussionswürdig. So kommentierte beispielsweise die Neue Zürcher Zeitung (NZZ) die Durchführung der Pressekonferenz erst rund einen Monat nach dem Unfall mit den Worten: „die seit Wochen über Seveso schwebende ,Wolke‘ von Gerüchten und Halbwahrheiten [ist] - endlich möchte man sagen - an mehreren Stellen durchstoßen und der Blick auf Fakten freigegeben worden.“ Die Stellungnahme der $N Z Z$ verdeutlicht die Wirkung der kommunikativen Zurückhaltung. Sie produziere, so die genutzte Metapher, eine zweite Wolke. Neben der Aerosolwolke des Unglücks entstehe eine Wolke der Vermutungen. Dies verdeutlicht, inwiefern das Schweigen als folgenreiche Form der Kommunikation verstanden wurde. Die fehlende Positionierung des Unternehmens wurde 1976 in den Medien des Mainstreams und nicht mehr wie noch 1921 vor allem in der linken Parteipresse als Versuch des Vertuschens interpretiert. ${ }^{71}$ Der isolierte Rekurs auf den Patriarchalismus als Steuerungssystem war 1976 gesellschaftlich kaum noch anschlussfähig und wirkte in weiten Kreisen der Medien, aber auch der Bevölkerung, wie der Zulauf zu sozialen Bewegungen verdeutlicht, delegitimierend.

Dazu trug auch bei, dass die praktizierte Form der patriarchalen Unternehmenskommunikation beinahe anachronistisch war. Obwohl Hoffmann-La Roche über einen Pressesprecher verfügte, war die Außenkommunikation ganz auf die Personen des Präsidenten bzw. anderer Entscheider auf Direktorenebene zuge-

69 Ebda., S. 252, wie Fußnote 34.

70 Berghoff, Unternehmenskultur, S. 203.

71 Vgl. exemplarisch die Titelgestaltung auf jener Seite des Blicks, auf der das Interview mit Jann abgedruckt ist: Das Interview, oben auf der Seite positioniert, ist mit den Worten „was der Hoffroche-Boss sagt...“ überschrieben. Ihm folgt ein Artikel mit der ebenso großen Überschrift „....und wie es für die Opfer von Seveso wirklich ist“, der den Worten Janns das Schicksal zweier von Chlorakne betroffener Kinder, der Geschwister Alice und Stefanie Senno, entgegen stellt. Blick, Hoffroche-Boss. 
schnitten. Sie folgte dem überholten „Prinzip der verschlossenen Tür und der zusammengekniffenen Lippen “" ${ }^{72}$ Dies begrenzte die kommunikativen Möglichkeiten des Unternehmens: Zum einen vernachlässigte es eine kontinuierliche Öffentlichkeitsarbeit, wie sie Public Relations-Konzepte, die seit den 1950er Jahren auch europäische Unternehmenspolitiken prägten, vorsah. „Ziel der Public Relation (PR) war es, das unternehmerische Ansehen in der Öffentlichkeit, etwa durch Pressearbeit, soziales Engagement und die Kultivierung einer, corporate personality', zu fördern und auf diese Weise identitätsstiftend zu wirken." ${ }^{\text {"73 }}$ Indem Hoffmann-La Roche darauf verzichtete, wurde gleichsam versäumt, eine Grundlage für die aktive Kommunikation im Krisenfall, wie er infolge des Unfalls eintrat, zu schaffen. Dies galt nicht nur für den Aufbau von Beziehungen zu den Anspruchsgruppen des Unternehmens, die zentral für die Legitimation unternehmerischen Handelns sind, sondern auch für die Professionalisierung der Unternehmenskommunikation. Der Unfall machte deutlich, dass kein geeignetes Konzept für die Kommunikation mit einer Transparenz und Partizipation fordernden Medienöffentlichkeit vorlag. Zum anderen ignorierte das Unternehmen relevante kommunikationswissenschaftliche Entwicklungen, wie eben jene von Paul Watzlawick Anfang der 1960er Jahre formulierte Überlegung, dass jegliches Verhalten Kommunikation sei. Es erstaunt nicht, dass in einem solchen Wissenskontext die von Hoffmann-La Roche geübte kommunikative Zurückhaltung angesichts des Unfalls von der Medienöffentlichkeit als Botschaft des Verschweigens und Vertuschens interpretiert wurde.

\section{Inhaltliche Schwerpunktsetzungen unternehmerischer Kommunikation}

Das Kommunikationsverhalten von Carl Bosch und Adolf W. Jann unmittelbar nach dem Unfall ist nicht nur durch formale Aspekte wie die Terminierung, die Kommunikationsanlässe, -formen und -adressaten gekennzeichnet, sondern auch durch inhaltliche Schwerpunktsetzungen. Es waren vor allem vier Themenfelder,

72 A. Schmidt, Public Relations als unternehmerische Aufgabe in der Neuen und der Alten Welt, Heidelberg 1959, S. 60.

73 S. Hilger, „Amerikanisierung“ deutscher Unternehmen. Wettbewerbsstrategien und Unternehmenspolitik bei Henkel, Siemens und Daimler-Benz (1945/49-1975), Stuttgart 2004, S. 259, hier in Bezug auf ein Werk von Anfang der 1950er Jahre, das für die Durchsetzung des Konzeptes wichtig war: C. Hundhausen, Werbung um öffentliches Vertrauen (public relations), Essen 1951. 
welche die Stellungnahmen auf Seiten der Unternehmensleitungen strukturierten: Selbstverortung, Unfallhergang und damit unmittelbar verknüpft Verantwortung und Zukunftsperspektiven. Sie werden im Folgenden hinsichtlich ihrer sprachlichen und argumentativen Gestaltung vergleichend analysiert und dabei die letzten drei Aspekte in enger Verflechtung miteinander besprochen.

1921 begann Carl Bosch seine Rede bei der Trauerfeier für die Opfer des Explosionsunglücks vier Tage nach dem Unfall unmittelbar mit einer Selbstverortung: „Mit schwerem Herzen trete ich heute vor Sie hin im Auftrage des Vorstandes der Anilinfabrik, von der die erschütternde Katastrophe ausging, um deren Opfern heute die letzte Ehre zu erweisen. “74 Die Zielgruppenansprache ist offensichtlich. Vergleichbar der Reaktion unmittelbar nach dem Unfall, als Bosch mit Lokalpolitikern und leitenden Angestellten sprach, adressierte er mit seiner Rede eine dritte relevante Anspruchsgruppe des Unternehmens - die Opfer. Er tat dies auch im Format der Rede erneut persönlich, indem er die Ich-Form nutzte und seine Ausführungen mit einem Verweis auf seinen individuellen emotionalen Gemütszustand begann. ${ }^{75}$ Diese persönlichen Bezüge führte der Vorstandsvorsitzende der BASF weiter aus: Er definierte sich als „Erbauer des Oppauer Werkes“: „Doppelt schwer wird es mir selbst, [...] weil es mein Lebenswerk betrifft, an dem ich mit allen Fasern meines Herzens hänge, dessen Werdegang ich von Anfang an miterlebt habe." ${ }^{76}$ Kommunikativ wurde dem Oppauer Werk auf diese Weise eine herausgehobene Stellung in der Berufsbiographie Carl Boschs zugeschrieben. Aus dieser resultierte, das wird impliziert, eine besondere Sorge um und für das Werk. Dieser Aspekt wird im weiteren Verlauf der Rede auf die Frage nach der unternehmerischen Verantwortung für den Unfall und einer möglicher Zukunftsgestaltung übertragen, wie noch zu zeigen sein wird. Doch die Herausstellung des Werkes hat eine weitere Facette. Carl Bosch personalisierte es rhetorisch. Das Werk erhielt gewissermaßen einen Lebenslauf, der von der Planung über die Erbauung bis hin zu seinem später dargestellten volkswirtschaftlichen Beitrag und schließlich seiner Zerstörung im Unfall reichte. Damit kam es zu einem Rollenwechsel: Das Werk wurde vom Ort des Geschehens zum Opfer desselben.

74 Werkszeitung, Rede, S. 139. An dieser Stelle sei auf die semantische Konstruktion hingewiesen, die offen lässt, wer die Opfer verursacht hat - die Anilinfabrik oder die Katastrophe. Die Reihung jedoch legt den Bezug auf das Wort Katastrophe nahe.

75 Dies wird nur eingeschränkt durch den Verweis auf den Auftrag des Vorstandes. Er bindet die exponierte Stellung Boschs ein und verteilt damit Verantwortlichkeit rhetorisch auf mehrere Schultern.

76 Werkszeitung, Rede, S. 139. 
Auch Adolf W. Jann verortete sich in seinen Stellungnahmen 1976 persönlich. In seiner ersten öffentlichen Äußerung, dem Interview mit dem Blick, tat er dies an zwei Stellen. Angesprochen auf die Gefahren der chemischen Industrie antwortete er: „Ich bedauere den Vorfall. Aber man muss eben auch die Gefahren, die ein chemischer Betrieb mit sich bringt, in Rechnung stellen. Sonst müsste man ja auf der ganzen Welt die Chemie einstellen.“ Zuvor führte er bereits hinsichtlich des Engagements der Konzernleitung vor Ort aus: „Ich habe ausdrücklich Weisung erteilt, dass Dr. Waldvogel (Givaudan-Direktor) nicht hinuntergeht. Aber unsere Experten sind an Ort und Stelle, ebenso neutrale Fachleute. “77 Vergleichbar äußerte er sich auf der Pressekonferenz am 11. August, die er mit einem tiefen Bedauern der Unternehmensleitung begann, um dann auf das vom Unternehmen zusammengestellte Expertenteam hinzuweisen. ${ }^{78}$ Es wird deutlich, dass Adolf W. Jann die Selbstverortung nutzte, um zum einen den eigenen Leitungsanspruch und damit zugleich die hierarchische, patriarchale Ordnung des Konzerns öffentlich zu kommunizieren. Er versuchte auf diese Weise das Integrationspotential, das einem geteilten Ordnungskonzept innewohnt (was der Patriarchalismus allerdings 1976 nicht mehr war) kommunikativ zu mobilisieren. Zum anderen positionierte Jann in seinen Ausführungen das Unternehmen als Akteur. Dies geschah durch Einordnung - in den Kontext der chemischen Industrie allgemein ebenso wie in das Feld der handelnden Personen vor Ort. Sprachlich wurde das Geschehen relativiert, wenn dem Bedauern der Verweis auf weltweite Gefahren chemischer Produktion folgte. Das verwendete aber schränkte den Ausdruck des Bedauerns ein. Zugleich wurde durch die Betonung der eigenen unternehmerischen Entscheidungskompetenz und ihrer Richtung Dr. Waldvogel geht nicht hinunter - das patriarchalische Verständnis, dass der Platz des Unternehmers das Unternehmen sei, transportiert. Infolge dieser Legitimationsfunktion der Selbstverortung gerieten die Anspruchsgruppen des Unternehmens aus dem Blick. Sie fanden sprachlich keine Berücksichtigung. Dennoch wurde die im Patriarchalismus ebenfalls wichtige Fürsorgefunktion des Unternehmens nicht ignoriert. Vielmehr sollte der Verweis auf Experten sie indirekt kommunizieren.

Der Vergleich zeigt, dass die Personalisierung ein wesentliches Merkmal der Selbstverortung ist. Diese bezieht sich keineswegs nur darauf, eine Relation zwischen dem Sprechendem und dem Ereignis herzustellen. Vielmehr kann sie

77 Beide Zitate aus Blick, Hoffroche-Boss.

78 Neue Zürcher Zeitung, Hoffmann-La Roche. 
auch genutzt werden, um agency neu zu perspektivieren. ${ }^{79}$ Carl Bosch tat dies im Hinblick auf das Werk, das sprachlich gewissermaßen belebt wurde. Auf diese Weise konnte Verantwortung delegiert werden. Das Werk, eigentlich Verursacher des Unfalls, wurde zu seinem Opfer, was zugleich das Unternehmen entschuldete. Adolf W. Jann nutzte die Personalisierung in ähnlicher, wenn auch konventionellerer Weise, wenn er Experten und neutralen Fachleuten Handlungsmacht zusprach und damit den Blick weg vom Verursacher hin zur Schadensbekämpfung lenkte.

Damit sind zugleich wesentliche Merkmale der Kommunikation von Verantwortung durch Adolf W. Jann benannt. Jann rekurrierte auf eine Funktionstrennung zwischen der Unternehmensleitung, die Steuerungsaufgaben zu erfüllen habe, und den von ihr bestellten Fachleuten, deren Aufgabe die Untersuchung des Unfallhergangs vor Ort und die Bewältigung der Unfallfolgen sei. ${ }^{80}$ Sachlich durchaus gerechtfertigt, wird hier deutlich, wie sehr die Steuerungsfunktion des Patriarchalismus betont wurde. Über die Aufgabenteilung hinaus lassen sich noch weitere Elemente eines funktionslogischen Argumentationsaufbaus beim Präsidenten von Hoffmann-La Roche nachweisen. Auch die Zusicherung von Schadensersatzleistungen, eine inhaltlich weitgehende medienöffentliche Positionierung des Präsidenten von Hoffmann-La Roche, folgte dieser Logik. Jann betonte die Deckung der Kosten durch Versicherungen. ${ }^{81}$ Die Medien interpretierten dies im Sinne fehlender Empathie. So kommentierte der Spiegel: „Aber noch drei Wochen nach der Katastrophe suchte Adolf W. Jann [...] das Unglück herunterzuspielen. Es sei ein, wenn auch bedauerlicher ,Vorfall‘, gegen dessen materielle Folgen man ,auch versichert' sei. Die Menschen in Seveso sahen es anders. ,Unser kleines Hiroshima“ nannte es Vittorio Rivolta, oberster Gesundheitsbeamter der Region." 82 Diese Wahrnehmung überdeckte die inhaltliche Aussage von Jann, materiell Verantwortung zu übernehmen. ${ }^{83}$

79 Zum Konzept der agency vgl. D. Bakan, The Duality of human Existence: An Essay on Psychology and Religion, Chicago 1966, S. 16.

80 Vgl. auch die Wiedergabe der Worte Janns auf der Pressekonferenz in der Neuen Zürcher Zeitung, Hoffmann-La Roche: „Man habe die besten Leute im ganzen Konzern in einem ,CrashProgramm ' vereinigt, um der Katastrophe Herr zu werden, das heißt, um die ausgetretenen Giftstoffe zu neutralisieren und zu vernichten. [...] Roche habe sich die Mitarbeit der besten Experten auf diesem Gebiet gesichert.“

$\mathbf{8 1}$ „Wir kommen selbstverständlich für alle Schäden auf [...]. Dafür sind wir auch versichert.“, in: Blick, Hoffroche-Boss.

82 Spiegel, Geplünderte Erde, S. 120.

83 Wobei es auch andere Interpretationen des Gesagten gab, wie das Ende des Berichts der Neuen Zürcher Zeitung über die Pressekonferenz deutlich macht. Neue Zürcher Zeitung, Hoffmann-La Roche. 
Vergleicht man diese Form der medialen Rezeption von Äußerungen der Unternehmensleitung zur Verantwortung für das Geschehen mit jener aus dem Jahr 1921, so fällt ein Unterschied unmittelbar ins Auge. Carl Bosch machte in seiner Rede auf der Trauerfeier keinerlei Zusagen. Vielmehr betonte er vor jeder eingehenden Untersuchung des Unfallhergangs, dass das Unternehmen keine Verantwortung für das Geschehen trage: „Kein Kunstfehler und keine Unterlassungssünde hat die Katastrophe herbeigeführt. Neue, uns auch jetzt noch unerklärliche Eigenschaften der Natur haben all unseren Bemühungen gespottet.“ In diesem Sinne bot Carl Bosch in seiner Rede „nur das Mitgefühl“ an und den „Dank für das, was die Toten uns waren, dem ich hiermit im Auftrage des Vorstands und des Aufsichtsrates tiefgefühlten Ausdruck gebe““. ${ }^{84}$ In den bürgerlichen Medien traf diese vorwegnehmende Bewertung nicht auf Kritik, sie wurde nicht einmal näher kommentiert. Dazu trug sicher auch bei, dass sie sich in der Nutzung der Naturmetapher zur Beschreibung der Unfallursachen mit den Ausführungen von Reichspräsident Friedrich Ebert während der Trauerfeier deckte. Auch er sprach von „unbekannten Kräften der Zerstörung“, die in eine „Musteranstalt deutschen Unternehmergeistes“ eingedrungen seien. ${ }^{85}$ Beide rekurrierten damit auf ein Narrativ des Fortschrittsdenkens, nach dem der Mensch der Natur durch ihre wissenschaftlich-technische Durchdringung gesellschaftliche Wohlfahrt unter Opfern abtrotzt. Das Unglück wurde in dieser Interpretation zu einem kaum vorhersehbaren Schicksalsschlag - und mehr als das: Es wurde zu einer unhintergehbaren Konsequenz des „Kampf[es] der Menschheit mit den Naturkräften“, der ausgefochten werden müsse: „selbst heute, vor den offenen Gräbern zwingt uns das unerbittliche Muß bereits wieder auf den Weg weiterer Pflichterfüllung. “86 Zwar blieben solche Narrative 1921 nicht mehr unhinterfragt, doch trugen sie weiter das Versprechen gesellschaftlicher Wohlfahrt durch technisch-industrielle Produktion und eben Pflichterfüllung in sich.

So wie das Werk und mit ihm das Unternehmen durch die Personalisierung entschuldet wurde, sollte die Naturalisierung eine Verlagerung von Verantwortung für das Unfallgeschehen befördern. Nicht die Produktion, so die Logik des Narrativs, sei Auslöser der Explosion, sondern eine nicht vollständig beherrschte Natur. Sicherheit und damit weitere gesellschaftliche Wohlfahrt, so die Botschaft, könne nur durch eine weitere Durchdringung der Natur mit wissenschaftlichtechnischen Mitteln erlangt werden. Auch die Naturalisierung diente damit der

84 Beide Zitate in Werkszeitung, Rede, S. 139.

85 Rede Friedrich Eberts bei der Trauerfeier für Opfer des Explosionsunglücks am 21.09.1921, abgedruckt in: Werkzeitung der Badischen Anilin- \& Soda-Fabrik Ludwigshafen 10, 1921, S. 140.

86 Werkszeitung, Rede, S. 139 f. 
Entschuldung des Unternehmens, das nichts falsch gemacht habe, sondern nur in seinen Bemühungen, die Natur zu beherrschen nicht gut genug gewesen sei. Diese Entschuldung sollte indirekt auch ausbleibende materielle Zusagen legitimieren. ${ }^{87}$ Die Metapher unerklärliche Eigenschaften der Natur trug 1976 angesichts einer gesellschaftlich bereits verankerten Fortschrittskritik nicht mehr und Adolf W. Jann bemühte sie auch gar nicht erst. Vielmehr argumentierte er ökonomisch-rational, wenn er die Aufgabentrennung zwischen wirtschaftlich verantwortlicher Unternehmensleitung und medizinischen oder toxikologischen Fachleuten betonte oder auf die Deckung der anfallenden Schadensersatzkosten durch Versicherungen hinwies. Angesichts einer fehlenden Vertrauensbeziehung zwischen Unternehmen und Medienöffentlichkeit konnte er die damit intendierte Seriosität auf diese Weise jedoch nicht vermitteln.

Mit seiner Perspektivierung der Verantwortung für das Unfallgeschehen hatte Carl Bosch zugleich auch Stellung zum Unfallhergang genommen. Durch den Bezug auf die unerklärlichen Eigenschaften der Natur wurde der industrielle Unfall einer Naturkatastrophe gleichgesetzt. Der Einfluss des technisch-industriellen Komplexes auf das Geschehen wurde auf diese Weise sprachlich überformt und der Natur und mit ihr dem Produkt ein Akteursstatus zugeschrieben. „Gerade der Stoff, der bestimmt war, Millionen unseres Vaterlandes Nahrung zu schaffen [...], hat sich plötzlich als grimmiger Feind erwiesen. [...] Unser Werk hat er in Schutt und Asche gelegt. “ ${ }^{88}$ Auf diese Weise wurde Verantwortung verlagert ohne die eigene Expertise infrage zu stellen, da diese die Grundlage einer zukünftigen besseren Durchdringung der „letzten Geheimnisse“ der Natur versprach. ${ }^{89}$ Vor dieser Folie konnte Carl Bosch in der Trauerrede auch die Vision eines Wiederaufbaus des Unglücksbetriebs und damit eine Zukunftsperspektive entwickeln. Indem er das Oppauer Werk als den Ort stilisierte, an dem sich dank der dortigen Produktion von Kunstdünger der Kampf um die „Erhaltung unseres Vaterlandes“ entschied, betonte er den Gestaltungsanspruch und die Kompetenz der BASF. ${ }^{90}$ Diese reichten in einer historisch längeren Perspektive über die Nahrungsmittelversorgung hinaus. Auch im Zweiten Weltkrieg kam der Stickstoffproduktion wie bereits 1914 bis 1918 große militärische Bedeutung zu.

Die Stellungnahme Adolf W. Janns zum Unfallhergang unterschied sich von der Carl Boschs, obwohl auch Jann zunächst ein Nichtwissen hinsichtlich des

87 Vgl. K. Patzel-Mattern, Krisen und Unfälle: Institutionen zwischen Beharrung und Lernen, in: Dies./C. Wischermann/M. Lutz/T. Jungkind (Hg.), Studienbuch institutionelle Wirtschaftsund Unternehmensgeschichte, Stuttgart 2015, S. 240-257, hier S. 240 f.

88 Beide Zitate Werkszeitung, Rede, S. 139.

89 Ebda.

90 Ebda. 
genauen Unfallhergangs betonte. Waren es bei Bosch die unerklärlichen Eigenschaften der Natur, so verwies Jann auf „einen technischen Vorfall, den wir uns nicht erklären können.“ Im Gegensatz zu 1921 konnte 1976 die Natur nicht mehr als Gegenspieler des Menschen in Stellung gebracht werden. Vielmehr erschien diese - der Rekurs auf Rachel Carsons Roman im Spiegel macht dies deutlich selbst als Opfer der Industrie. Vor diesem Hintergrund öffnete sich ein Erklärungsvakuum, alte Legitimationsstrategien erwiesen sich als nicht mehr mobilisierbar. Ein rationaler Ansatz, wie ihn Guy Waldvogel, Generaldirektor der Givaudan, bei der Pressekonferenz anbot, verdeutlicht dieses entstandene Erklärungsvakuum: „Im Laboratorium nachträglich unternommene Versuche, den Unfall im Kleinmaßstab nachzuvollziehen, blieben bisher erfolglos. Es kommt somit auch beim heutigen, fortgeschrittenen Stand der Untersuchungen noch immer sowohl technisches als auch menschliches Versagen als Auslösefaktor in Frage. " ${ }^{\text {"91 }}$ Angesichts der eingestandenen Unkenntnis der genauen Ursachen des Unfalls trotz ihrer wissenschaftlichen Untersuchung auch mehr als einen Monat nach dem Ereignis, konnte das Unternehmen nur schwer seine Kompetenz für die Gestaltung zukünftiger Produktion vermitteln und so Perspektiven entwickeln. Vielmehr erschien es rat- und visionslos - so auch Adolf W. Jann in einem Blick-Interview auf die Frage nach dem möglichen Ende der Dioxin-Produktion: „Zuerst muss nun alles abgeklärt werden. Wir hoffen, wir bringen es heraus. "92 Das Versagen des industriell-technischen Komplexes im Unfall sowie die Unmöglichkeit einen alternativen, unternehmensexternen Akteur des Geschehens aufzubauen, stellten unternehmerische Handlungskompetenzen infrage. Diese unterschiedlichen, kommunikativ vermittelten Ansprüche auf Zukunftsgestaltung spiegelten sich auch in der materiellen Bewältigung der Unfallfolgen: Oppau blieb als Produktionsstätte mit unmittelbarer Nachbarschaft von Werk und Ort bestehen, während in Meda das Werk geschlossen und in Seveso die Industrielandschaft durch eine Renaturierung überschrieben wurde.

\section{Von der Unmöglichkeit nicht zu kommunizieren. Ein Fazit}

Der Vergleich des kommunikativen Krisenmanagements von Carl Bosch und Adolf W. Jann verweist auf die Relevanz, die Leitbildern für gelingende unterneh-

91 Neue Zürcher Zeitung, Hoffmann-La Roche.

92 Blick, Hoffroche-Boss. 
merische Kommunikation nach industriellen Unfällen zukommt. Zwar fußten die Stellungnahmen beider Unternehmensleitungen auf einem patriarchalen Verständnis, doch wurden diesem 1921 und 1976 unterschiedliche Funktionen zugeschrieben. Während der Patriarchalismus 1921 noch als wechselseitige Beziehung von Unternehmensleitung und Mitarbeitern verstanden wurde und, wenn auch mit Einschränkungen, gesellschaftlich akzeptiert war, erschien er 55 Jahre später einer kritischen Medienöffentlichkeit als anachronistisches Steuerungsinstrument der Unternehmenskommunikation. Im Sinne einer hierarchischen, der Einbeziehung von Öffentlichkeiten in unternehmerische Entscheidungsprozesse skeptisch gegenüberstehenden Kommunikationspolitik wurde das Festhalten an tradierten, patriarchalischen Formen der Kommunikation zum Auslöser eines Delegitimationsprozesses des Handelns von Hoffmann-La Roche. Dies wird anhand der, aus unterschiedlichen Interpretationen des Patriarchalismus erwachsenden, zeitlichen Differenzen in der Kommunikation der Unternehmensleitungen deutlich. Während Carl Bosch unmittelbar nach dem Ereignis reagierte, dauerte es fast einen Monat, bis Adolf W. Jann sich öffentlich äußerte. Auch intern kommunizierten Entscheider des Basler Unternehmens auf der Leitungsebene erst mit einwöchiger Verzögerung und agierten damit anders als Bosch es für die BASF tat. Es ist gerade diese zeitlich verzögerte Kommunikation, die dazu führte, dass Hoffmann-La Roche Gestaltungkompetenz einbüßte. Das Schweigen wurde als Stellungnahme - sei es als Vertuschungsversuch, sei es als Orientierungslosigkeit - interpretiert; und dieser Eindruck wurde durch inhaltliche Aspekte verstärkt. Carl Bosch bot 1921 mit den unerklärlichen Eigenschaften der Natur schon wenige Tage nach dem Unglück eine Erklärung für das Unfallgeschehen an. Sie erschien angesichts eines gesellschaftlich akzeptierten Fortschrittsnarrativs mit einer zu erkämpfenden Unterordnung der Natur unter die Bedürfnisse des Menschen plausibel. Die genutzte Metapher konnte Bindekraft entwickeln und die Beziehung zwischen dem Unternehmen und seinen Anspruchsgruppen stärken. Demgegenüber konnte Adolf W. Jann auch rund einen Monat nach dem Dioxinaustritt im Jahr 1976 noch keine definitiven Unfallursachen benennen. Wissenschaftliche Untersuchungen, auf die sich das Unternehmen berief, erschienen in einer Gesellschaft, in der sich eine Skepsis gegenüber den Potentialen der technischen Durchdringung der Natur etabliert hatte, nur noch begrenzt tauglich, um Lösungskompetenz zu kommunizieren. Vor dem Hintergrund, dass Hoffmann-La Roche es versäumt hatte, eine Vertrauensbeziehung zur Medienöffentlichkeit aufzubauen, wurde der Bezug auf ökonomische und technische Rationalität nicht als Ausweis von Solidität des Unternehmens und seiner Leitung, sondern als fehlende Empathie wahrgenommen. Gelingende Kommunikation nach industriellen Unfällen, aber auch die Akzeptanz von Schweigen in einzelnen Punkten, so lässt sich abschließend fest- 
halten, basiert auf weitgehend geteilten Leitbildern. Sie sind die Grundlage für die gesellschaftliche Legitimierung unternehmerischen Handelns im Krisenfall.

\section{Bionote}

\section{Katja Patzel-Mattern}

Professorin für Wirtschaft und Sozialgeschichte an der Ruprechts-Karls Universität Heidelberg, Studium der Neueren und Neuesten Geschichte, Publizistik und Politikwissenschaft an der Westfälischen Wilhelms-Universität Münster und der Universidad de Barcelona, Promotion an der Universität Münster (1998), Habilitation an der Universität Konstanz (2008). Aktuelle Projekte zur industriellen Krisenkommunikation, zur „Humanisierung des Arbeitslebens”, zur Aneignung von Stadträumen in Zeiten soziopolitischer Umbrüche und zur Geschichte frühkindlicher Betreuung; Publikationen u.a. zu industriellen Katastrophen und Krisen, zur Gestaltung von Arbeitsbeziehungen und zu kulturwissenschaftlicher Theoriebildung. 